\title{
Bubbling grape juice
}

$\mathrm{T}$ his Practice Corner illustrates how busy general practitioner (GPs) can use a journal club to keep up to date with the best current research, to support care decisions for individual patients, to communicate with researchers about questions for which no satisfactory evidence exists, and to improve our daily clinical work.

\section{CLINICAL SCENARIO}

A 58 year old man was diagnosed with a $7 \times 6 \mathrm{~cm}$ liver tumor on ultrasound (US) after presenting with abdominal distension for 1 week. He was a chronic hepatitis B carrier, detected 3 years ago in an insurance health check. Yearly blood tests have been performed since then. Four months ago, both alanine transaminase (ALT) and alpha feto protein $(\mathrm{AFP})$ were in the normal range $(\mathrm{ALT}=35 \mathrm{IU} / \mathrm{l}$; $\mathrm{AFP}=1.25 \mathrm{ng} / \mathrm{ml})$.

Subsequently, left hepatectomy was performed with a pathology report of hepatocellular carcinoma (HCC) TNM Stage III. In the letter, the surgeon estimated his 5 year survival rate to be $25 \%$ according to local data. His wife, daughter, and son are asymptomatic hepatitis B carriers with normal ALT. The wife and daughter are HBe Ag positive while the son is HBe Ag negative. Understandably, they are all very concerned about the risk of having HCC in the future, and they wish to be "checked" and "treated earlier."

\section{CLINICAL QUESTION}

Working in an endemic area for chronic hepatitis B, this scenario is not uncommon. The Hong Kong Cancer Registry in 2000 showed 1584 new cases and 1424 deaths from HCC. Lifetime risk was 1 in 30 for men and 1 in 110 for women.

Our question was: in asymptomatic chronic hepatitis B carriers, will screening by AFP and/or liver US reduce mortality from liver cancer?

\section{SEARCH STRATEGY \& APPLICATION OF THE EVIDENCE}

A relevant Cochrane systematic review was retrieved using the terms chronic hepatitis $B$, screening, and liver cancer. We were amazed at the paucity of controlled trials. The systematic review was done in a rigorous way, but the rate limiting step in EBM seems to lie at the research level.

Based on the evidence ( see CAT below), we could give our patients this information:

\footnotetext{
About 5000 patients need to have a blood test and ultrasound every 6 months to reduce 1 death from liver cancer in 1 year. It costs about US $\$ 120$ per year. There is the possibility of detecting the cancer when it is smaller, hence surgery could be an option, after which one might live 6 months longer. Our knowledge is still limited; the hope to "catch the disease" earlier and hence live longer is not based on firm evidence.
}

CAT: SCREENING OF LIVER CANCER BY AFP AND/ OR US IN CHRONIC HEPATITIS B MAY BE EFFECTIVE Clinical bottom line:

1. AFP and US performed every 6 months in patients with chronic hepatitis B result in a reduction in HCC mortality, but the difference is not significant. (OR 0.81, CI 0.54 to 1.22 , NNS $=937 / 5 \mathrm{y})$.

2. Screening appears to detect more HCC in 5 years (but not so when updated to $7 \mathrm{y}$ follow up).

3. Screening significantly detects more resectable HCC $(<5$ cm) (OR 7.14, CI 3.53 to 14.43).

4. 5 year survival rate after resection in the screened group is longer, with lead time 5.4 months.

\section{Search terms in Cochrane library:}

chronic hepatitis B, screening, liver cancer

Reference: Wun YT, Dickinson JA. Alpha-fetoprotein and / or liver ultrasonography for liver cancer screening in patients with chronic hepatitis B. Cochrane Database Syst Rev 2003;(2):CD002799.

\section{The study:}

1. The systematic review: randomised trials on screening for liver cancer irrespective of language. Of the 550 journal abstracts, only 2 trials met the selection criteria (!)

2. Shanghai trial (Yang 1999): 18816 randomised to screening by biannual AFP plus US (9372) or control (9443), followed up for 5 years. Outcomes include HCC mortality, number of HCC detected, HCC resectability, and mortality of resected patients. Personal communication provides additional information equivalent to a 7 year follow up.

3. Toronto trial (Sherman 1995): 1069 randomised to screening by AFP (531) or screening by AFP plus US (538), followed up for 26 months. Sample size not adequate to compare the 2 screening methods.

\section{The evidence}

See table 1 .

\section{Comments:}

1. Not enough quality trials to support or refute screening. People recruited for screening should be informed that to date "any screening performed more in hope of benefit than with certainty."

2. Marked difference in cost of screening in different locality. Quoted as US $\$ 0.60$ per AFP and US $\$ 1.20$ for each ultrasound. In Hong Kong, the corresponding cost is about USD $\$ 40$ and $\$ 20$, respectively. A difference of $\sim 70$ times.

3. No data on $\mathrm{NNH}$, eg. anxiety if result is falsely positive. Appraised by: Amy Chan /JASPA* Feb 2004.

\section{HOW WE LEARN TOGETHER}

Our journal club has 5 GPs working in different sectors: government, group practice, and solo practice. We have used the 3 part agenda depicted in Sackett's book (ie, discussing clinical problems and formulating them in an answerable 
Table 1 The evidence (mainly based on Shanghai study)

\begin{tabular}{lllll}
\hline Outcome & Screened AFP + US & Unscreened & OR (95\% CI) & NNS \\
\hline HCC mortality & $0.44 \%$ & $0.54 \%$ & $0.81(0.54$ to 1.22$)$ & $937 / 5 \mathrm{y}$ \\
HCC detected & $0.92 \%$ & $0.54 \%$ & $1.69(1.20$ to 2.36$)$ & $693 / 5 \mathrm{y}$ \\
HCC resectability $(<5 \mathrm{~cm})$ & $0.42 \%$ & $0 \%$ & $7.14(3.53$ to 14.43$)$ & \\
5 year survival after HCC resection & $84.9 \%$ & $0 \%$ & & \\
\hline
\end{tabular}

$\mathrm{Cl}=$ confidence interval; $\mathrm{HCC}=$ hepatocellular carcinoma; NNS = number needed to screen; $\mathrm{OR}=$ odds ratio.

PICO (patient, intervention, comparison, and outcome) format, searching and photocopying articles, critical appraisal of a selected piece of evidence worth studying (the beef), and assigning people to write up a "critically appraised topic" (CAT). In addition, we also spare some time to brainstorm, "buzz," and "fish bowl" one another on such issues as patients' preferences and values and clinical circumstances.

For the above scenario, we role played and learnt, by multiple attempts, the best way to explain the evidence to our patients in simple terms. We also raised queries among ourselves from the patients' perspectives. It is during this process that many new questions arose and thereby uncovered our knowledge gap.

For example:

"Will I have a higher chance of developing HCC if I am HBe Ag positive?"

"What should I do if my liver enzymes are raised, even though the AFP \& US are normal?"

"I have heard of the drug lamivudine; should I take that? Will the drug cure the disease?"

"Is it safe to try herbal medicine?"

Striving for closure by the end of every session is antithetical, so we usually leave plenty of issues to reflect upon between sessions. Instead of having educational prescriptions stuffed in whitecoat pockets during clinical rounds (GPs seldom have ward rounds!) and tossing them in a tray, we circulate our clinical questions online. In the interim period, we can take a look at our "Bank of questions" (see table 2), and vote on the next question that we think is worth our time and effort. In this way, we make sure that the next topic is of interest to the group and that ample time is allowed for preparing the relevant articles.

\section{THE NEXT MOMENTUM}

By searching PubMed using Hepatitis B, chronic[MeSH] and limiting to "Practice Guidelines," 2 relevant guidelines were retrieved: the Asian-Pacific Consensus Statement published in 2003, and another one by the American Association of Study of Liver Disease (AASLD) published in 2001. Although published 3 years ago, we decided that the AASLD one is more evidence-based. While surfing Google in search of other existing guidelines, the 2004 International Liver Congress coorganised by AASLD was noted. We decided 1 member could attend the congress and share the latest evidence online (see the letter below). We planned to critically appraise the latest guideline published in March 2004. Still, our baskets of unanswered questions were bothering us so much that we decided to pose our burning queries to the researchers via the website of the Congress, hoping (remotely) to generate a pragmatic agenda for future health research.

\section{HOW WE PLAN TO MOVE ON}

As seen in our form for voting (table 2), a long queue of questions await answers. We plan to alternate our problem-based learning with sessions for prepared discussion: members will share their search on their individual educational prescriptions that could be answered swiftly on one's own. We hope that by doing so, we can incorporate deep learning with broad learning.

Meanwhile, we are circulating many of our findings to other colleagues on the internet. Of course, we also circulate our secret roster- "Who Is going to bring bubbling grape juice for the next session?" Perhaps this is the reason we attracted visitors to our last 2 meetings!

\section{Letter sharing learning points in a congress}

Dear all,

I attended the HK-Shanghai International Liver Congress 2004 and here are some learning points:

(1) Experts from 3 continents (USA, Europe, Asia) all started their presentation with one statement: management must be INDIVIDUALISED. There can be no hard and fast rule or any global consensus, just a balance of evidence and resources.

(2) Both the AASLD (American Association for the Study of Liver Disease) and the EASL (European Association for the Study of the Liver) have guidelines developed under a rigorous process. The boards (or jury) are mainly expert hepatologists in the AASLD; while for EASL, independent other-field doctors are also involved. The guideline drafted by the APASL (Asian Pacific Association for the Study of the Liver) seems to be least evidence-based, but they have considered more local issues - for example, the use of herbal medicine and the YMDD mutations, which are seen more often in the Asian Pacific region.

(3) $\mathrm{HBeAg}$ positive and $\mathrm{HBeAg}$ negative patients are considered 2 separate diseases: the current practice is to use interferon more in the $\mathrm{e}+\mathrm{ve}$, and oral anti-virals (lamivudine/adefovir) more in the e -ve. Another consensus is to wait for longer $(6 \mathrm{mo})$ if a flare up occurs in an e+ve before embarking on treatment.

The other focus is "who are the candidates that might benefit from treatment", or "whom should be referred to the hepatologists for a liver biopsy?"

But these questions keep ringing in my mind:

i) What is the relationship of e antigen status to the development of HCC?

ii) It seems quite clear to the hepatologists whom/how they are going to "treat", but based on what evidence should GPs monitor our asymptomatic HB carriers with normal LFT? How often are we to check the ALT? 3 months? 6 months? Do we just go by the pockets of our patients? Are there any subgroups that worth a closer monitoring?

iii) Screening for HCC, well, sorry, no one mentioned this.

I had no chance to raise these questions in the meeting-too many other "specialist questions." Anyway, any comments? Amy 


\section{CONCLUSION}

Having a group is cost effective: it helps to stimulate and maintain our interest and enthusiasm, and makes the practice of EBM faster, easier, and merrier. We hope you will enjoy your time as well.
${ }^{*}$ JASPA is a scoring system for measuring personal angst towards medical literature; see BMJ 1995;311:1666-8.

AMY CHAN, MBBS (HK), FRACGP, FHKCFP, FOR THE JASPA GROUP Hong Kong

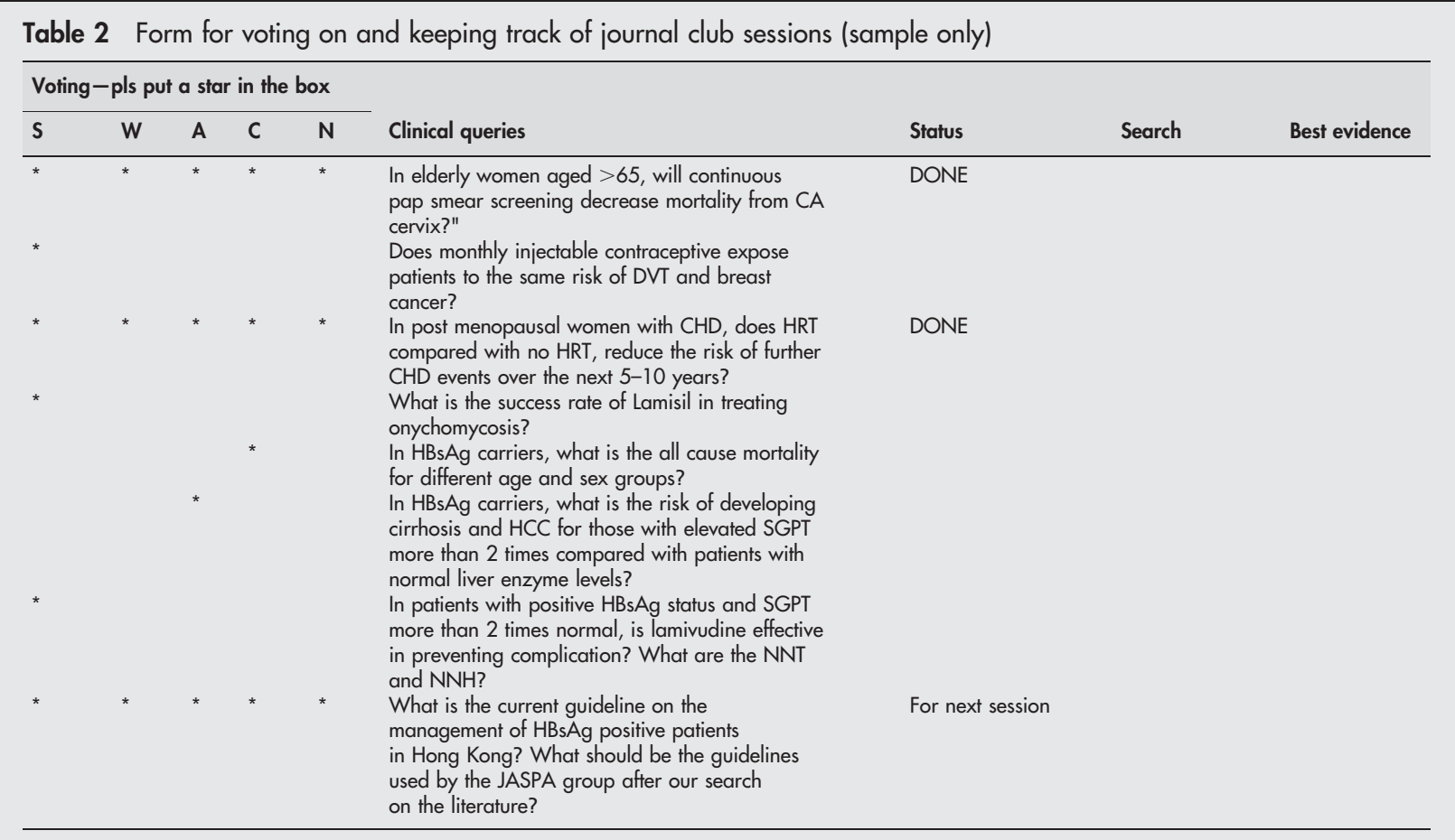

\section{$11^{\text {th }}$ UK Workshop in Teaching Evidence-Based Practice.}

$\mathrm{T}$ he annual workshop at the Centre for Evidence-Based Medicine (CEBM) in Oxford is designed to explore and practice different ways of teaching evidence-based practice. After 11 years of workshops the CEBM, through generous donations, was this year able to offer bursaries to several individuals from developing countries. Competition was fierce for places with 36 applicants for 7 places. This year's bursaries enabled folk to attend from Africa, Asia, and Uzebekistan. The latter was a GP trainer who established an Evidence-Based Centre in Uzbekistan in 2004, the goal of which is to promote EBM throughout the country and to aid implementation of research findings in practice. If you are interested in a place for next year, please check the CEBM website (www.cebm.net) in January.

The format of the workshop remains similar to the one David Sackett instigated at the $1^{\text {st }}$ UK Workshop in Oxford. Over the years, the workshop has been refined in response to participant feedback. The workshop is mostly small group work, plus a daily plenary session, and is used to discuss general issues in planning, executing, and evaluating, as well as demonstrating large group strategies for teaching EBM.
For the past 2 years each small group has had its own librarian facilitator for the week, which participants greatly value.

The 2 main themes of the workshop are teaching and personal development. Teaching is addressed through learning and trying out different educational models for teaching evidence-based practice and discussion of the strategies, techniques, and approaches that teachers can use to facilitate learning, as well as issues of curriculum design, development, and maintenance. Personal Development is addressed by offering guidance and help in extending participants' existing critical appraisal and teaching skills.

The CEBM is pleased to announce an agreement with CASP International Network to bring together the CEBM workshop and the CASP International Train the Trainer Week next year on $11-15^{\text {th }}$ September 2006 at St Hugh's College (see www.cebm.net).

EMMA MEATS, MSC Centre for Evidence-Based Medicine, Oxford University Oxford, UK 